his visit to the Cameroon Mountains, and his ascent of the river Binué.

THE Bolletino of the Italian Geographical Society for April contains full details of the proposed Arctic Expedition under Lieut. Bove, with a carefully compiled map of the, sonth polar regions so far as these liave been hitherto explored.

M. Desire Charnay bas left New York for Mexico for the purpose of carrying out a thorough exploration of the ancient remains that still exist in that country. It is expected that the work of exploration will last for two or three years.

\section{ON ELECTRIC LIGHTING}

D YNAMO-ELECTRIC MACHINES. - Since the date of the author's former paper in April, 1879, other observers have published the results of experiments similar to those described by him. It may be well to exhibit some of these results reduced to the form he has adopted, viz., a curve, such as that shown in Fig. 4, Proceidings, 1879, Plate 29, and now reproduced, with slight alterations, in Fig. I. Here any abscissa represents a current passing through the dynamo-electric machine,

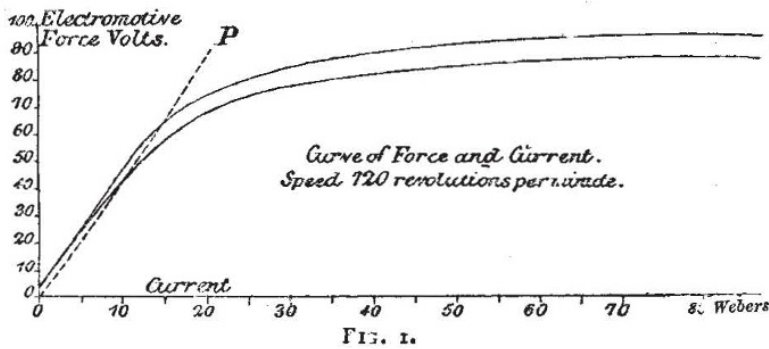

and the corresfonding ordinate represcnts the electromotive force of the machine for a certain speed of revolution, when that current is passing through it. It will be found (I) that with varying speed the ordinate or electromotive force, corresponding to any abscissa or current, is proportional to the speed ; $(2)$ that the electromotive force does not increase indefinitely with increasing current, but that the curve approaches an asymptote ; (3) that the earlier part of the curve is, roughly speaking, a straight line, until the current attains a certain value, and that at that point the electromotive force has reached about two-thirds of its maximum value. When the current is such that the electromotive force is not more than two-thirds of its maximum, a very small change in the resistance with speed of engine constant, or in the speed of the engine with resistance constant, causes a great change in the current. For this reason such a current, which is the same for all speeds of revolution, since the curves for different speeds differ only in the scale of ordinates, may be called the "critical current" of the machine. The effect of a change of speed is exhibited in Fig. I, where the low er line represents a curve for a speed of 660 revolutions per minute, instead of 720 . The resis'ance, varying as electromotive force is given by the slope of the line $O P$, which must therefore be constant; and it will be seen that this line cuts the upper curve at a point corresponding to a current of 15 webers, and the lower at a point corresponding to a current of 5 webers only.

In Germany, Auerbach and Meyer (Wiedemann's Annalen, November, 1879) have experimented fully on a Gramme machine at various speeds, and with various external resistances. The resistance of the machine was $0^{\circ} 97$ ohms. Their results are summarised in a table at the end of their paper, which gives the current passing, with resistances in circuit from $r 75$ to 200 Siemens units, and at speeds from 20 to 800 revolutions per minute. In the accompanying diagram, Fig. 2, curve No. I, ex presses the relation between electromotive force and current, as deduced from some of their observations, making allowance, where necessary, for difference in speed. The curve, as aciually constructed, is for a speed of 800 revolutions : at this speed it will be seen that the maximum electromotive force is about 76 volts the critical current, corresponding to a force of about $5 \mathrm{I}$ volts, is 6.5 webers, with a total resistance of $7.8 \mathrm{ohms}$. Up to this point there will be great instability, exactly as was the case in

X Paper read at the Institution of Mechanical Engineers, by Dr. Jobn Hopkinson, F.R.S. the Siemens machine examined by the author, where the resistance was 4 ohms, and the speed 720 revolutions.

The results of an elaborate series of experiments on certain dynamo-electric machines have recently been presented to the Royal Society by Dr. Siemens. One of the machines examined was an ordinary medium-sized machine, substantially similar to that tried by the author in 1879 . It is described as having 24 divisions of the commutator ; 336 coils on the armature, with a resistance of 0.4014 Siemens units; and 512 coils on the mag. nets, with a resistance of 0.3065 ; making a total resistance of

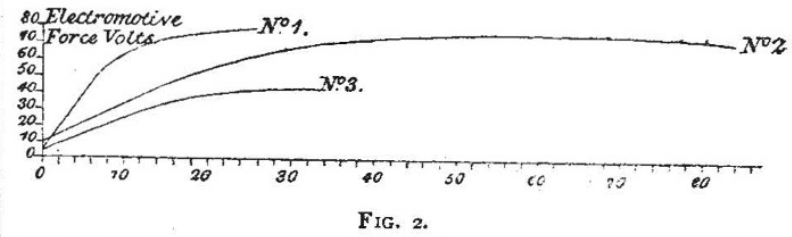

0.7079 Siemens units $=0.6654$ ohms. Curve No. 2 gives the relation of electromotive force and current, reduced to a speed of 700 revolutions per minute, the actual speeds ranging from 450 to 800 revolutions. The maximum electromotive force appears to be probably 76 volts, and the critical current 15 webers, which is the same as in the author's first experiments on a similar machine.

In the summer of last year the author examined a Siemens machine of the smallest size. This machine is generally sold as an exciter for their alternate current macbine. It has an internal resistance of $0.74 \mathrm{ohms}$, of which 0.395 is in the armature or helix. The machine is marked to run at 1,130 revolutions per minute. The following Table gives, for a speed of 1,000 revolutions, the total resi-tance, current, electromotive force, and horse-power developed as current. The horse-porer expended was not determined :-

Experiments on smallest-sized Siemens Dynamo-Electric Machine

\begin{tabular}{|c|c|c|c|c|c|c|c|c|c|}
\hline \multicolumn{2}{|l|}{ Resistance. } & \multicolumn{3}{|c|}{$\begin{array}{l}\text { Electric } \\
\text { current. } \\
\text { Webers. }\end{array}$} & \multicolumn{3}{|c|}{$\begin{array}{l}\text { Electromotive } \\
\text { force. } \\
\text { Volts. }\end{array}$} & \multicolumn{2}{|c|}{$\begin{array}{l}\text { Horse-power deve- } \\
\text { loped as current. } \\
\text { H. P. }\end{array}$} \\
\hline Onms. & $\ldots$ & $\ldots$ & 4.53 & $\cdots$ & $\ldots$ & $13 \cdot 2$ & $\ldots$ & & 0.08 \\
\hline $2 \cdot 221$ & $\ldots$ & $\ldots$ & I0.8 & $\cdots$ & $\cdots$ & 270 & $\ldots$ & $\ldots$ & 0.39 \\
\hline$I \cdot 967$ & $\ldots$ & $\ldots$ & $15^{\circ} 1$ & $\ldots$ & $\ldots$ & $33^{\circ} 6$ & $\ldots$ & $\ldots$ & \\
\hline 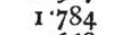 & $\ldots$ & $\cdots$ & 18 & $\cdots$ & $\ldots$ & 36.4 & $\ldots$ & $\ldots$ & 0.88 \\
\hline 8 & $\ldots$ & $\ldots$ & & $\ldots$ & $\ldots$ & $37^{\circ} \cdot 2$ & $\ldots$ & $\ldots$ & 0.98 \\
\hline 9 & $\ldots$ & $\cdots$ & 20 & $\ldots$ & $\ldots$ & & $\ldots$ & $\ldots$ & I OI \\
\hline $1 \cdot 503$ & $\ldots$ & $\ldots$ & 22.8 & $\ldots$ & $\ldots$ & 39.3 & $\ldots$ & $\ldots$ & $I^{\prime} 20$ \\
\hline & $\ldots$ & $\ldots$ & 24 & $\ldots$ & $\ldots$ & $40^{\circ} 0$ & $\ldots$ & $\ldots$ & $r \cdot 32$ \\
\hline$x \cdot 145$ & $\ldots$ & $\ldots$ & $32 \cdot 2$ & $\ldots$ & $\ldots$ & $41 \cdot 5$ & $\ldots$ & $\ldots$ & $I^{*} 79$ \\
\hline
\end{tabular}

Curve No. 3 gives as usual the relations of electromotive force and current. From this curve it will be seen that the critical current is $\mathbf{I} \mathbf{I}^{\cdot} \mathbf{2}$ webers, and the maximum electro. motive force, at the speed of $x, 000$ revolutions, is about 42 volts. The determinations for this machine were made in exactly the same manner as in the experiments on the medium-sized machine, using the galvan meter, but omitting the experiment with the calorimeter (compare Table I., p. 249, Proceedings, April, I879).

The time required to develop the current in a Gramme macbine has been examined by Herwig (Wiedemann, June, 1879). He established the following facts for the machine he examined. A reversed current, having an electromotive force of $0^{\circ} 9$ Grove cells, sufficed to destroy the residual magnetism of the electromagnets. If the residual magnetism was as far as possible reduced, it took a much longer time to get up the current than when the machine was in its usual state. A longer time was required to get up the current when the external resistance was great, than when it was small. With ordinary resistance the current required from $\frac{3}{4}$ second to $\mathrm{I}$ second to attain its maximum. Brightness of the Electric Arc.- The measurement of the light emitted by an electric arc presents certain peculiar difficulties. The light itself is of a different colour from that of a standard candle, in terms of which it is usual to express luminous intensities. The statement, without qualification, that a certain electric lamp and machine give a light of a specified number of candles, is therefore wanting in definite meaning. A red light cannot with propriety be said to be any particular multiple of a green light ; nor can one light, which is a mixture of colours, be said with strictness to be a multiple of another, unless the proportions of the colours in the two cases are the same. Ca t. Abney (Proceedings of the Rryal Society, March, 1878) has given the 
results of measurements of the red, blue, and actinic light of electric arcs, in terms of the red, blue, and actinic light of a standard candle. The fact that the electric light is a very different mixture of rays from the light of gas or of a candle, has long been known, but has been ignured in statements intended for practical purposes.

Ayain, the emission of rays from the heated carbons and arc is by no means the same in all directions. Determinations bave been made in Paris of the intensity in different directions, in particular cnses. If the measurement is made in a horiz'ntal

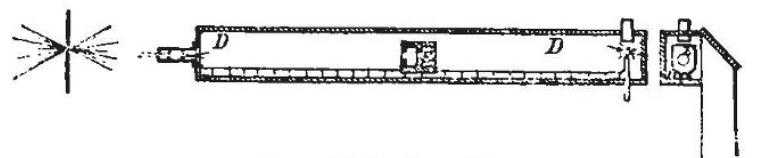

Fig. 3 ! Scale about $\left.{ }_{60}^{\top}\right)$.

direction, a very small obliquity in the crater of the positive carbon will throw the light much more on one side than on the other, causing great discordance in the results obtained.

If the electric light be compared directly with a standard candle, a dark chamber of great length is needed-a convenience not alway: attainable. In the experiments made at the South Foreland by Dr. Tyndall and Mr. Douglase, an intermediate standard was employed; the electric light was measured in terms of a large oil lamp, and this latter was frequently compared with a standard candle.

Other engagements have prevented the author from fairly attacking the e difficulties; but since May 1879 he has had in occasional use a photometer with which powerful lights can be measured in moderate space. This photometer is shown in Fig. 3, and an enlargement of the field-piece in Fig. 4. A lens

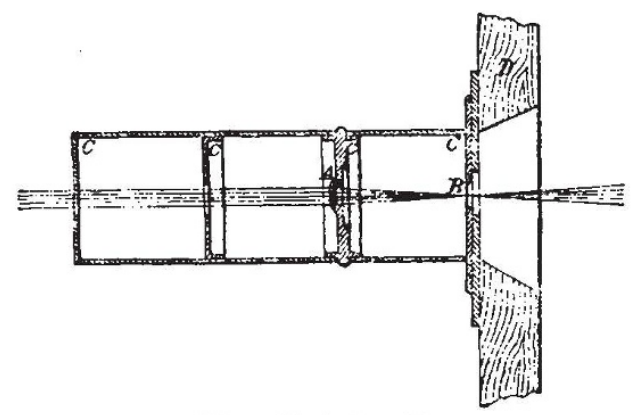

Fic. \& (Scale ab ut 1$)$.

A, of short focus, forms an image at B of the powerful source of light which it is desired to examine. The intensity of the liuht from this image will be les; than that of the actual source by a calculable amount; and when the distance of the lens from the light is suitable, the reduction is such that the reduced light becomes com parable with a candle or a carcel lamp. Diaphragms $\mathrm{CC}$ are arranged in the cell which contains the lens, to cut of stray light. One of these is placed at the focus of the lens, and has a small aperture. It is easy to see that this diaphragm will cut off all light entering from a direction other than that of the source ; so effectually does it do so, that observations may be made in broad daylight on any scurce of light, if a dark screen be placed behind it. The long b; D D, Fig. 3, of about 7 feet length, is lined with velvet--the old-fashioned dull velvet--not that now sold with a finish, which reflects a great desl of the light incident at a certain angle. This box serves as a dark chamber, in which the intensity of the image furmed by the lens $i$ : compared with a standard light, by mean; of an ordinary Bunsen's photometer E, sliding oin a graduated bar.

Mr. Dallneyer kindly had the lens made for the author: he can therefure rely $u_{i}$ )on the accuracy of it curvature and thickness; it is plano-convex, the $\mathrm{c}$ invex side being towards the source of light. The curvature is exactly $\mathbf{I}$ inch radius, and the thickness is 0.04 inch; it is made of Chance's hard crown glass, of which the refractive index for the $D$ line in the spectrum is 1.517. The focal length $f$ is therefore 1.933 inch.

Let $u$ denote the distance of the source of light froin the curved surface of the lens, and $v$ the di:tance of the image of the source B from the posterior focal plane. Neglecting for the moment loss by reflection at the surface of the glass, the intensity of the source is reduced by the factor $\left(\frac{v}{u}\right)^{2}$. But $\frac{\mathbf{I}}{v}+\frac{\mathbf{I}}{u}=\frac{\mathbf{I}}{f}$, or $v=-\frac{u f}{u-f ;}$ hence the factor of reduction is $(-f-f)^{2}$. The effect of absorption in so small a thickness of very pure glass may be neglected; but the reflection at the surfaces will cause a loss of $8^{\circ} 3$ per cent. which must be allowed for. This percentage is calculated from Fresnel's formulx, which are certainly accurate for glasses of moderate refrangibility, and for moderate angles of incidence.

Suppose, for example, it is required to measure a light of 8,000 candles; if it be placed at a distance of 40 inches it will be reduced in the ratio 467 to 1 , and becomes a conveniently measurable quantity. By transmitting through coloured glasses both the light from an electric lamp and that from the standard, a rough comparison may be made of the red or green in the electric light with the red or green in the standard.

A disjersive photometer, in which a lens is used in a somewhat similar manner, is described in Stevenson's "Lighthouse Illumination." Messrs. Ayrton and Perry described a dispersive photometer with a concave lens at the meeting of the Physical Society on December 13, 1879 (Proc. of the Physical Society, vol. iii. p. 184). The convex lens possesses however an obvious advantage in having a real $f$ cuss, at which a diaphragm to cut off stray light may be placed.

Efficiency of the Electric Arc. - To define the electrical condition of an electric arc, two quantities must be stated: the current passing, and the difference of electric potential at the ends of the two carbons. Instead of either one of these, we may, if we please, state the ratio $\frac{\text { difference of potential }}{\text { current }}$, and call it the resistance of the arc, that is to say, the resistance

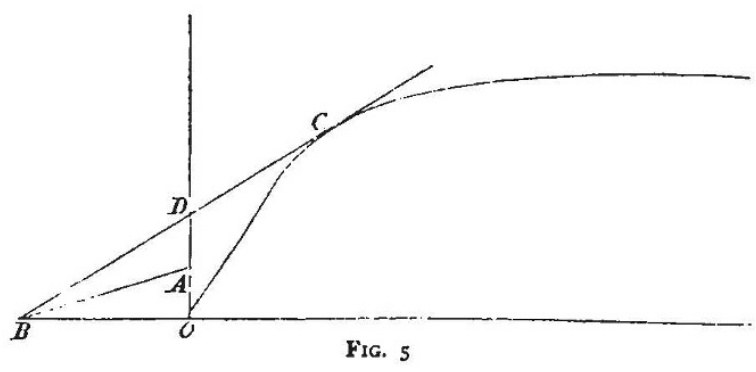

which w.uld replace the arc without changing the current. But such a use of the term electric resistance is unscientific; for Ohm's law, on which the definition of electric resistance rests, is quite untrue of the electric arc; and, on the nther hand, for a given material of the electrodes, a given distance between them, and a given atmospheric pressure, the difference of potential on the two sides of the arc is approximately constant. The product of the difference of potential and the current is of course equal to the work developed in the arc ; and this, divided by the work expended in driving the machine, may be considered as the efficiency of the whole combination. It is a very easy matter to measure these quantities. The difference of potential on the two sides of the arc may be measured by the method given by the author in his previous pajer, by an electrometer, or in other ways. The current may be meaured by an Obach's galvano. meter, by a suitable electro-dynamometer, or best of all, in the author's opinion, by passing the whole current, on its way to the arc, through a very small known resistance, which may be regarded as a shunt for a galvanometer of very high resistance, or to the circuit of which a very high resistance has been added.

It appears that with the ordinary carbons and at ordinary atmospheric pressure no arc can exist with a le:s difference of potential than about 20 volts; and that in ordinary work, with an arc about $\frac{1}{4}$ inch long, the d.fference of potential is from 30 to 50 volts. Assuming the former result, about 20 volts, for the difference of potential, the use of the curve of electromotive forces may be illustrated by determining the lowest speed at which a given machine can run, and yct be capable of producing a short arc. Taling $O$ as the origin of co-ordinates, Fig. 5, set off upon the axis of ordinates the distance $O A$ equal to 20 volts; draw $A$ B to intersect at $B$ the negative prolongation of the axis 
of absciss $x$, so that the ratio $O A$ may represent the necessary metallic resistance of the circuit. Through the point $\mathrm{B}$, thus obtained, draw a tangent to the curve, touching it at $\mathrm{C}$, and cutting $O A$ in $\mathrm{D}$. Then the speed of the machine, corresponding to the particular curve employed, must be diminished in the ratio $\frac{O D}{O A}$, in order that an exccedingly small arc may be just possible.

The curve may also be employed to put into a somewhat different form the explanation given by Dr. Sicmens at the Royal Socicty respecting the occasional instability of the electric light as produced by ordinary dynamo-electric machines. The operation of all ordinary regulators is to part the carbons when the current is greater than a certain amount, and to close them when it is less; initially the carbons are in contact. Through the origin 0 , Fig. 6 , draw the straight line $0 \mathrm{~A}$, inclined at the angle

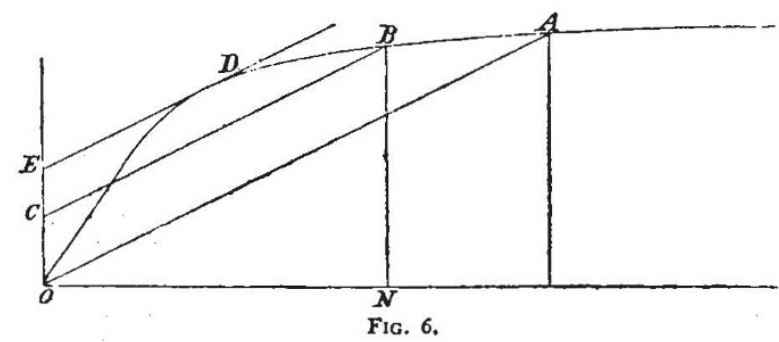

representing the resistances of the circuit other than the arc, and meeting the curve at $\mathrm{A}$. The abscissa of the point $\mathrm{A}$ represents the current which will pass if the lamp be prevented from operating. Let $\mathrm{ON}$ represent the current to which the lamp is adjusted; then if the abscissa of $A$ be greater than $O N$, the carbons will part. Through $\mathrm{N}$ draw the ordinate is $\mathrm{N}$, meeting the curve in the point $\mathrm{B}$; and parallel to $\mathrm{OA}$ draw a tangent $E D$, touching the curve at $D$. If the point $B$ is to the right of $D$, or farther from the origin, the arc will persist ; but if $B$ is to the left of 1 ), or nearer to the origin, the carbons will go on parting, till the current suddenly fails and the light goes out. If $B$, although to the right of $D$, is very near to it, a very small reduction in the speed of the machine will suffice to extinguish the light. Dr. Siemens gives greater stability to the light by exciting the electromagnets of the machine by a shunt circuit, instead of by the whole current.

The success of burning more than one regulating lamp in series depends on the use in the regulator of an electro-magnet, excited by a high-resistance wire connecting the two opposed carbons. The force of this magnet will depend upon the difference of potential in the arc, instead of depending, as in the ordinary lamp, upon the current passing. Such a shunt magnet has been employed in a variety of ways. The author has arranged it as an attachment to an ordinary regulator; the shunt magnet actuates a key, which short-circuits the magnet of the lamp when the carbons are too far parted, and so causes them to close.

In conclusion the author ventures to remind engineers of the following rule for determining the efficiency of any system of electric lighting in which the electric arc is used, the arc being neither exceptionally long nor exceptionally short. Measure the difference of potential of the arc, and also the current passing through it, in volts and webers respectively; then the product of these quantities, divided by 746 , is the horse-power developed in that arc. It is then known that the difference between the horse-power developed in the arc and the horse-power expended to drive the machine must be absolutely wasted, and has been expended in heating either the iron of the machine or the copper conducting wires.

PRELIMINARY REPORT B Y THE COMMITTEE ON SOLAR PHYSICS APPOINTED BY THE LORDS OF THE COMMITTEE OF COUNCIL ON EDUCATION

" $\mathrm{SIR},-$ In reply to $\mathrm{Mr}$. MacLeod's letter of November 20 , 1879, calling upon us, pending the preparation of our General Report, to give a brief summary of the progress already made, and to state at the same time what work was in hand, and such other facts as we might think it desirable to mention, to enable their Lordships to determine whether they shall apply to the Lords Commissioners of Her Majesty's Treasury for an extension of the vote for another year, we have the honour now to submit the following report.

"The Committee have had thirteen formal meetings. In addition to this several members of the Committee have carried out special branches of the inquiry; and Mr. Lockyer, as arranged when the Committee was appointed, has been charged with the general conduct of the observational and experimental work at South Kensington. The Committee consider that Mr. Lockyer by his laboratory work and comparison of the results with solar phenomena, has brought together a great body of evidence tending prima facie to conclusions of the utmost importance. The labour and difficulty of the research are, however, so great that much additional time and attention must continue to be bestowed on it before the questions thus raised can be considered as finally settled; and the Committee think it of much importance that the researches now being carried on should not be interrupted.

"The Committee have been in correspondence with the Indian Government, the Astronomer-Royal, the Directors of the Observatories at Wilna, Melbourne, Mauritius, Kew, Moscow, Toronto, Paris, Palermo, Princeton, and with Ir. Warren De la Rue. From all of these promises of valuable coroperation and assistance have been received. To the Astronomer-Royal our thanks are specially due for the manner in which he has met us in the matter, placing all the information bearing on the subject in the Royal Observatory at our service.

"A few months before the appointment of the Committee, daily photographs of the sun had been commenced by order of the Government of India under the Surveyor General at Dehra, N.W. Provinces, the photographs being transmitted to Mr. Lockyer for reduction.

"Unfortunately the observer, Mr. Meins, late of the R.E., sent from this country, after having been trained at Chatham and South Kensington, died suddenly in the early part of the year, and the continuity of the daily record was thus broken. In August the Government of India requested to be informed as to the importance of the continuance of the records thus interrupted, and the following letter was sent in reply :-

$$
\text { " 'Science and Art Department, London, S.W., }
$$

" ' SIR, - In reply to your letter dated August 10, 1879 , inviting remarks relative to the importance of continuing certain solar observations which were recently instituted in India, and suggestions as to future arrangements if it should be decided that the observations are to bc continued for an indefinite period, I beg leave to submit to you, for the information of Lord Cranbrook, the following explanation :-

" In their General Report the Science Commissioners recommended the establishment of a system of physical (as distingtished from astronomical) observations on the sun, and pointed out the advantages which Northern India offers for this study. A memorial was more recently presented to Government, signed by a number of our leading scientific men, urging the carrying out of this recommendation.

" IIn compliance with these recommendations the British Government as a preliminary step appointed a Committee on Solar Physics, whose duty it should be to make trial of methods of observation, collect observed results, \&c., and who were specially charged with the reduction of such observations as should. be made in India. As a consequence of this arrangement the Government of India authorised the employment of the late Mr. Meins for the purpose of taking photographs of the sun in India, and a series of such photographs was prepared by him and has been sent home to be dealt with. The following brief statement will show how superior the climate of India is to our own for observations of this kind. The Astronomer-Royal has been so kind as to furnish the Committee with a list of the solar photographs taken at the Royal Observatory during the period 21st July, 1873, to 18th July, 1879, over a part of which $\mathbf{M r}$. Meins' work extended. It should be mentioned that in both places alike the rule was to take three photographs daily, in the morning, about noon, in the afternoon, when clear views. of the sun could be obtained. In the rare cases in which a fourth photograph was taken in the same day in India, it is. not included in the following list :- 